Article

\title{
Enaminones in Heterocyclic Synthesis: A Novel Route to Tetrahydropyrimidines, Dihydropyridines, Triacylbenzenes and Naphthofurans under Microwave Irradiation
}

\author{
Saleh Mohammed Al-Mousawi *, Morsy Ahmed El-Apasery and Mohamed H. Elnagdi \\ Department of Chemistry, Faculty of Science; Kuwait University, P.O. Box 5969 Safat, 13060, Kuwait \\ * Author to whom correspondence should be addressed; E-Mail: saleh.almousawi@yahoo.com; \\ Tel.: +965-24985547; Fax: +965-24816482.
}

Received: 27 October 2009; in revised form: 18 December 2009 / Accepted: 23 December 2009 / Published: 25 December 2009

\begin{abstract}
Condensation of phthalimidoacetone (1) with DMFDMA ( $N, N$ Dimethylformamide dimethyl acetal) has afforded enaminone 2. Refluxing 2 with equimolecular amounts of benzaldehyde and urea in acetic acid afforded a mixture of tetrahydropyrimidine $\mathbf{5}$ and the dihydropyridine $\mathbf{6}$. Compound $\mathbf{2}$ undergoes selfcondensation on heating in acetic acid or under microwave irradiation in presence of acidic zeolite to give 1,3,5-triacylbenzene 9. Reacting enaminone 11a with naphthoquinone 15 afforded the naphthofuran 18. The possible formation of the aldehyde 19 was excluded based on an HMQC experiment, which revealed that the carbonyl carbon is not linked to any hydrogen.
\end{abstract}

Keywords: enaminones; Biginelli's condensation; naphthofuran; zeolites; microwave irradiation with simultaneous cooling

\section{Introduction}

Enaminones are valuable intermediates in synthetic organic chemistry [1-4]. In the last few years we and others have reported a variety of syntheses of heteroaromatics that have been developed using functionally substituted enamines as readily obtainable building blocks possessing multi electrophilic and nucleophilic moieties [5-9]. In conjunction to this work we report here an efficient microwave assisted synthesis of 2-(4-dimethylamino-2-oxo-but-3-enyl)isoindole-1,3-dione (2) and the results of 
our investigations devoted to exploring its synthetic potential. The work has enabled new synthetic approaches to the title compounds. We report here on the utility of 2 as precursor to substituted naphthofurans. In the course of this research we observed that the enaminone reactions were rather sluggish at room temperature, affording the final naphthofuran in moderate yield., but during the course of our explorations, we observed that the rate and the yields of these reactions could be greatly improved when performed under microwave irradiation in combination with simultaneous cooling at $30{ }^{\circ} \mathrm{C}$. In this contribution, we would like to disclose the preliminary results of our investigations.

\section{Results and Discussion}

Condensation of phthalimidoacetone (1) with dimethylformamide dimethyl acetal (DMFDMA) has afforded enaminone 2 in $76 \%$ and $77 \%$ yields, respectively, either by refluxing in xylene for $8 \mathrm{~h}$ or by heating in MW without solvent at $180{ }^{\circ} \mathrm{C}$ for $20 \mathrm{~min}$. The same condensation product had been obtained earlier by Al-Mousawi et al. [10,11]. Fischer et al. [12] have used coupling of carbonyl carbons with protons to establish relative geometry extensively, but using ${ }^{13} \mathrm{C}$-NMR to identify the long range coupling of $\mathrm{C}-\mathrm{H}$ as an indication of the stereochemistry from the $\mathrm{CO}$ group is very difficult because of the appearance of carbonyl carbons as broad signals. This is expected theoretically because this carbonyl carbon has three different $2 J, 2 J$ and $3 J$ couplings with nearby protons. We have run NOE difference experimentx to establish the stereo-orientation of either $E$ or $Z$-forms for enaminone 2. The NOE showed that irradiating the alkene $\mathrm{CH}$ at $\delta 5.04 \mathrm{ppm}$ enhanced the dimethylamino protons at $\delta$ 2.79 and $3.08 \mathrm{ppm}$, confirming that they are proximal in space as required by the $E$-form ( $c f$. Scheme 1).

Scheme 1. Preparation of 2-(4-dimethylamino-2-oxo-but-3-enyl)isoindole-1,3-dione (2).
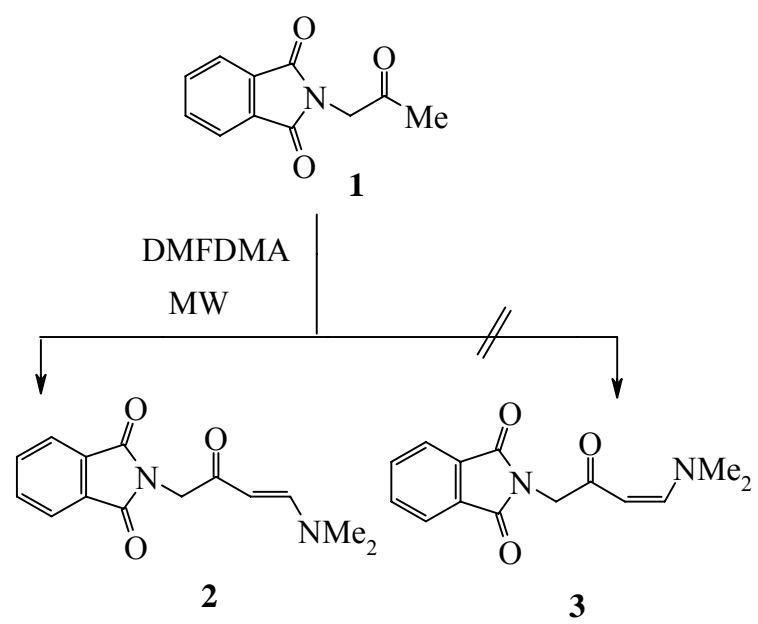

Compound 2, thus formed, has proven to be a versatile starting material for a variety of otherwise not readily obtainable functionally substituted aromatics and heteroaromatics. Thus, refluxing 2 with equimolecular amounts of benzaldehyde and urea in acetic acid afforded a 3:1 mixture of tetrahydropyrimidine 5 and the dihydropyridine $\mathbf{6}$, which was readily separated by fractional crystallization ( $c f$. Scheme 2). 
Scheme 2. Preparation of tetrahydropyrimidine 5 and dihydropyridine 6.<smiles>CN(C)C=CC(=O)CN1C(=O)c2ccccc2C1=O</smiles>
PhCHO / Urea $\mathrm{AcOH}$ 2<smiles>O=C(CN1C(=O)c2ccccc2C1=O)C1=CNC=C(C(=O)CN2C(=O)c3ccccc3C2=O)C1c1ccccc1</smiles>
6<smiles>CCCCC=C(C(=O)CN1C(=O)c2ccccc2C1=O)C(NC)NC(N)=O</smiles>

4<smiles>O=C1NC=C(C(=O)CN2C(=O)c3ccccc3C2=O)C(c2ccccc2)N1</smiles>

5

Compound 2 undergoes self-condensation upon heating in acetic acid for 10 hours or upon irradiation at $150{ }^{\circ} \mathrm{C}$ for 1 hour in a focused microwave oven in the presence of an acidic zeolite (Montmorillonite K-10) to give 1,3,5-triacylbenzene 9. We believe that compound 2 is initially converted to the open chain intermediate 7 that further adds to one molecule of enaminone 2 to yield the intermediate 8, which aromatize under these reaction conditions to give the final isolable product $\mathbf{9}$ (cf. Scheme 3).

Scheme 3. Synthesis of 2-(2-3,5-di[2-(1,3-dioxo-2,3-dihydro-1H-2-isoindolyl)acetyl]phenyl-2-oxoethyl)-1,3-isoindolinedione (9).

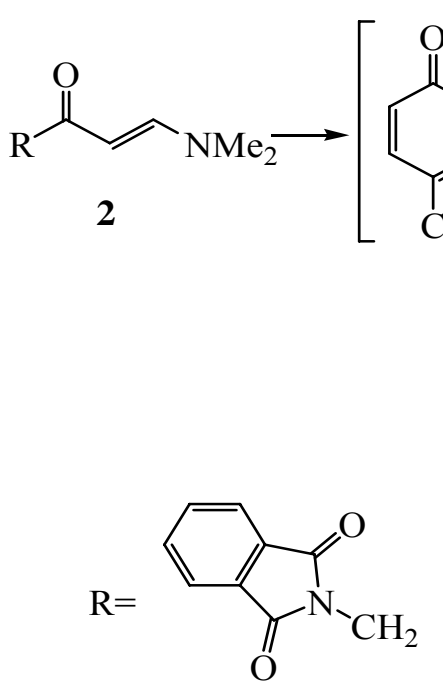<smiles>[R]C(=O)C1=CC(C([R])=O)C(N(C)C)C(C([R])=O)=C1</smiles>
$-\mathrm{HNMe}_{2}$<smiles>O=C(CN1C(=O)c2ccccc2C1=O)c1cc(C(=O)CN2C(=O)c3ccccc3C2=O)cc(C(=O)CN2C(=O)c3ccccc3C2=O)c1</smiles> 
Compound 2 reacted with anilines 10a-c to yield the aniline derivatives 11a-c. Compound 11a when heated with zeolite in an attempt to effect an intramolecular Friedel Craft's cyclization reaction under MW in the absence of solvent at $160{ }^{\circ} \mathrm{C}$ for 30 min afforded $N$-phenylphthalimide (12) that was readily obtained from the MW assisted reaction of phthalic anhydride (14) with aniline without solvent at $180{ }^{\circ} \mathrm{C}$ for $60 \mathrm{~min}$. It is most likely that in the initial step in this transformation the nitrogen atom lone pair attacks the phthalimidocarbonyl moiety. Similarly, heating (2) by MW in Zeolite without solvent at $160{ }^{\circ} \mathrm{C}$ for $30 \mathrm{~min}$. afforded $N$-methylphthalimide (13) (cf. Scheme 4).

Scheme 4. Synthesis of 2-(2-oxo-4-phenylamino-but-3-enyl)isoindole-1,3-dione, 2-[4-(4methoxyphenylamino)-2-oxo-but-3-enyl]isoindole-1,3-dione, and 2-[4-(4-nitrophenylamino)- 2-oxo-but-3-enyl]isoindole-1,3-dione.

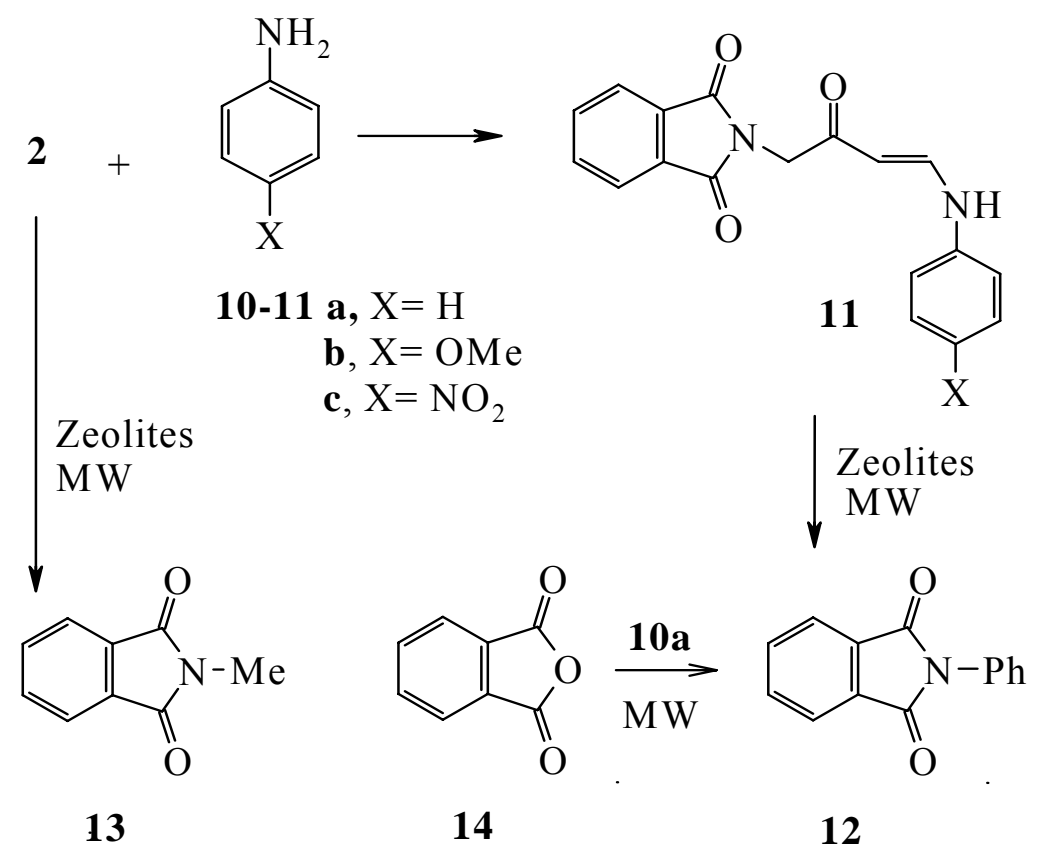

We decided to investigate the reaction utilizing microwave irradiation, while keeping the temperature at $30{ }^{\circ} \mathrm{C}$. This should also allow maintaining the maximum power input of $80 \mathrm{~W}$ during the full run of the irradiation. To ensure a correct temperature measurement, a fiber optic sensor was used. Using the same reagent ratios and solvent as those for the reaction run at room temperature $\left(\sim 25^{\circ} \mathrm{C}\right)$, we performed the reaction of enaminone 11a with naphthoquinone 15 under microwave irradiation with simultaneous cooling at $30^{\circ} \mathrm{C}$.

The reaction proceeded smoothly, furnishing the corresponding naphthofuran 18 . The yield of the product was increased to $76 \%$; The conventional method reaction at room temperature yielded only $68 \%$. It is noteworthy that the reactions could be performed using an irradiation power of $80 \mathrm{~W}$ continuously during the whole run, as the high power level was needed to maintain the temperature at $30{ }^{\circ} \mathrm{C}$ because of the efficient external cooling. The differences in yield could be attributed to a lower rate of decomposition of the compounds when using simultaneous cooling.

The possibility of the formation of the aldehyde 19 was excluded based on the HMQC data which revealed that the carbonyl carbon is not linked to any hydrogen. 
From $\mathrm{H}, \mathrm{H}-\mathrm{COSY}, \mathrm{H}-8$ at $\delta 7.70$ correlates with $\mathrm{H}-9$ at $\delta 8.23$ and $\mathrm{H}-7$ at $\delta 7.58$ correlates with $\mathrm{H}-8$ and with H-6 at $\delta 8.25 \mathrm{ppm}$. From HMBC, H-9 was detected at $\delta 8.23 \mathrm{ppm}$ because it correlates with C-9b at $\delta 145.0$, which also correlates with $\mathrm{H}-2$ at $\delta 9.31 \mathrm{ppm}$. CO-12 correlated at $\delta 167.99$ with $\mathrm{CH}_{2}-$ 11 at $\delta 5.70$ ppm and $\mathrm{CH}-13$ at $\delta 7.98$ ppm (cf. Scheme 5).

Scheme 5. Synthesis of 2-[2-(5-hydroxynaphtho[1,2-b]furan-3-yl)-2-oxoethyl]isoindole-1,3-dione.

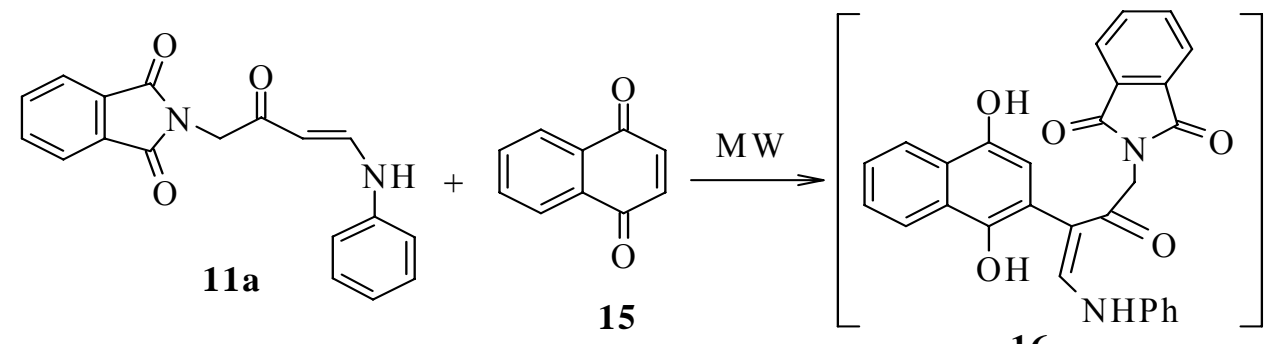

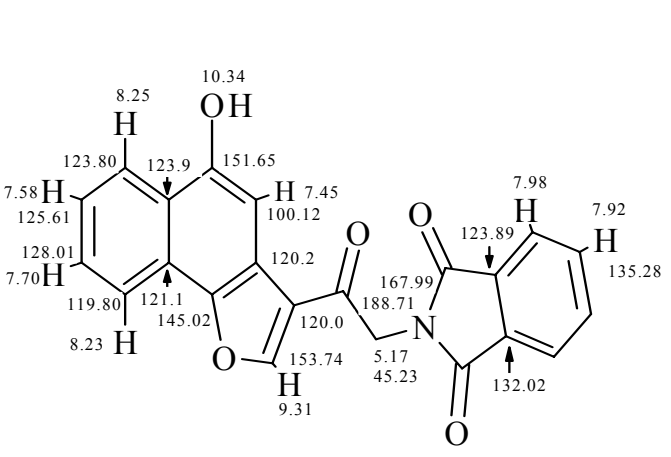

18<smiles>O=CC(C(=O)CN1C(=O)c2ccccc2C1=O)c1cc(O)c2ccccc2c1O</smiles>

17<smiles>O=Cc1c(CN2C(=O)c3ccccc3C2=O)oc2c1cc(O)c1ccccc12</smiles><smiles>C=CCC</smiles>

\section{Experimental}

\section{General}

Melting points are uncorrected. All the reactions were conducted under microwave irradiation in heavy-walled Pyrex tubes (capacity $10 \mathrm{~mL}$ ) fitted with PCS caps. Microwave heating was carried out with a single mode cavity Explorer Microwave Synthesizer (CEM Corporation, NC, USA), producing continuous irradiation and equipped with simultaneous external air-cooling system. Compound $\mathbf{1 7}$ was prepared in a microwave irradiation experiment which was carried out in a dedicated CEM-DiscoverCoolmate monomode microwave apparatus operating at a frequency of $2.45 \mathrm{GHz}$ with continuous irradiation power from 0 to $300 \mathrm{~W}$. The reaction was carried out in an open $10 \mathrm{~mL}$ double walled glass vial, which was cooled to $10{ }^{\circ} \mathrm{C}$ using a microwave transparent cooling liquid. The temperature was measured with a fiber-optic device inserted into the reaction vessel. IR spectra were recorded in $\mathrm{KBr}$ disks using a Perkin-Elmer System 2000 FT-IR spectrophotometer. ${ }^{1} \mathrm{H}-\mathrm{NMR}(400 \mathrm{MHz})$ and ${ }^{13} \mathrm{C}-\mathrm{NMR}(100 \mathrm{MHz})$ spectra were recorded on a Bruker DPX 400, super-conducting NMR 
spectrometer in $\mathrm{CDCl}_{3}$ or DMSO- $d_{6}$ as solvent and TMS as internal standard; chemical shifts were reported in $\delta$ units (ppm). Mass spectra were measured on a VG Autospec-Q (high resolution, high performance, tri-sector GC/MS/MS). Microanalyses were performed on a LECO CHNS-932 Elemental Analyzer.

\section{2-(4-Dimethylamino-2-oxo-but-3-enyl)isoindole-1,3-dione (2)}

Thermal method: A mixture of phthalimidoacetone $(2.03 \mathrm{~g}, 0.01 \mathrm{~mol})$ and DMFDMA (1.19 g, $0.01 \mathrm{~mol}$ ) in xylene $(5 \mathrm{~mL})$ was heated under reflux for $8 \mathrm{~h}$. The solid product was collected by filtration and crystallized from EtOH. The reaction gave yellow crystal, yield $(76 \%) ; \mathrm{mp} 162{ }^{\circ} \mathrm{C}$ (lit. [11] mp 159-162 $\left.{ }^{\circ} \mathrm{C}\right)$; IR (KBr) v 1,769, 1,714, 1,660 (CO) $\left(\mathrm{cm}^{-1}\right)$; MS (EI) m/z (\%): 258 [M $\left.{ }^{+}, 20 \%\right]$, 160(20), 76(10); ${ }^{1} \mathrm{H}-\mathrm{NMR}\left(\mathrm{DMSO}-d_{6}\right): \delta(\mathrm{ppm}) 2.72\left(\mathrm{~s}, 3 \mathrm{H}, \mathrm{CH}_{3}\right), 3.05\left(\mathrm{~s}, 3 \mathrm{H}, \mathrm{CH}_{3}\right), 4.40(\mathrm{~s}, 2 \mathrm{H}$, $\left.\mathrm{CH}_{2}\right), 5.04(\mathrm{~d}, 1 \mathrm{H}, J=12 \mathrm{~Hz}, \mathrm{CH}), 7.61(\mathrm{~d}, 1 \mathrm{H}, J=12 \mathrm{~Hz}, \mathrm{CH}), 7.85-7.91(\mathrm{~m}, 4 \mathrm{H}$, phthalimidyl-H); Anal. Calcd. for $\mathrm{C}_{14} \mathrm{H}_{14} \mathrm{~N}_{2} \mathrm{O}_{3}$ : C, 65.11; H, 5.46; N, 10.85; Found: C, 65.18; H, 5.44; N, 10.76 .

Microwave method: A mixture of phthalimidoacetone $(2.03 \mathrm{~g}, 0.01 \mathrm{~mol})$ and DMFDMA (1.19 g, $0.01 \mathrm{~mol}$ ) was irradiated by focused microwave at $180{ }^{\circ} \mathrm{C}$ for $20 \mathrm{~min}$. The build-up of pressure in the closed reaction vessel was carefully monitored. After the irradiation, the reaction tube was cooled with high-pressure air through an inbuilt system in the instrument until the temperature had fallen below $50{ }^{\circ} \mathrm{C}$. The solid product, so formed, was collected by filtration and crystallized from EtOH to give compound 2 in $77 \%$ yield.

\section{General procedure for the synthesis of compounds $\mathbf{5}$ and $\mathbf{6}$}

A solution of compound $2(2.58 \mathrm{~g} 0.01 \mathrm{~mol})$, benzaldehyde $(1.06 \mathrm{~g} 0.01 \mathrm{~mol})$, urea $(0.76 \mathrm{~g}$ $0.01 \mathrm{~mol})$, and acetic acid $(10 \mathrm{~mL})$ was heated under reflux for $3 \mathrm{~h}$ (completion of reaction was monitored by TLC). The precipitate thus formed was then collected by filtration, washed with water and crystallized from acetic acid to give (61\%) of tetrahydropyrimidine 5 . The filtrate was then washed thoroughly with water the solid product, so formed, was collected by filtration and crystallized from ethanol to afford dihydropyridine 6.

2-[2-Oxo-2-(2-oxo-4-phenyl-1,2,3,4-tetrahydropyrimidin-5-yl)ethyl]isoindole-1,3-dione (5): Yellow crystals, yield 61\%; mp 280-282 ${ }^{\circ} \mathrm{C}$; IR $\left(\mathrm{KBr}, \mathrm{cm}^{-1}\right): 3,236,3,101(\mathrm{NH}), 1,714,1,655$ (CO); MS (EI) m/z: (\%) 361 [M+, 15\%]; 342 (100), 316 (22), 200 (86), 172 (25). 157 (100), 131 (76), 103 (38), 77 (52); ${ }^{1} \mathrm{H}-\mathrm{NMR}\left(\mathrm{DMSO}-\mathrm{d}_{6}\right)$ : The diasterotopic protons $\mathrm{NCH}_{2}$ appear as two doublets $4.81,4.91(\mathrm{~d}, 1 \mathrm{H}$, $\left.J=13.6 \mathrm{~Hz}, \mathrm{~d}, 1 \mathrm{H}, J=13.6 \mathrm{~Hz}, \mathrm{NCH}_{2}\right), 5.15(\mathrm{~s}, 1 \mathrm{H}, \mathrm{CH}), 7.23(\mathrm{t}, 3 \mathrm{H}, J=8.0 \mathrm{~Hz}$, arom-H), 7.31 (t, $2 \mathrm{H}, J=7.2 \mathrm{~Hz}$, arom-H), 7.83-7.90 (m, 6H, arom-H, NH), $9.74(\mathrm{~s}, 1 \mathrm{H}, \mathrm{NH}) ;{ }^{13} \mathrm{C}-\mathrm{NMR}$ (DMSO- $\left.d_{6}\right)$ : $\delta=187.41,168.08,152.09$ (CO), 144.41, 140.92, 135.19, 132.80, 128.88, 127.84, 126.72, 123.70, 110.84, 53.20, $42.85\left(\mathrm{CH}_{2}\right)$; Anal. Calcd. for $\mathrm{C}_{20} \mathrm{H}_{15} \mathrm{~N}_{3} \mathrm{O}_{4}$ (361.35): C, 66.48; H, 4.18; N, 11.63. Found: C, 66.49; H, 4.40; N, 11.54.

2-[2-(5-(2-(1,3-Dioxoisoindolin-2-yl)acetyl)-4-phenyl-1,4-dihydropyridin-3-yl)-2oxoethyl] isoindoline-1,3-dione (6): Orange crystals, yield $20 \%$; mp 274-276 ${ }^{\circ} \mathrm{C}$; IR $\left(\mathrm{KBr}, \mathrm{cm}^{-1}\right)$ : 3,236, 3,101 (NH), 1,714, 1,655 (CO); MS (EI) m/z: (\%) 531 [M+, 14\%]; 513 (100), 454 (32), 369 
(55), 343 (22). 284 (18), 182 (26), 160 (100), 147 (72), 104 (74), 76 (61); ${ }^{1}$ H-NMR (DMSO-d ${ }_{6}$ ): 4.76-4.90 (m, 4H, 2NCH 2$), 5.16(\mathrm{~s}, 1 \mathrm{H}, \mathrm{CH}), 7.10(\mathrm{t}, 3 \mathrm{H}, J=7.6 \mathrm{~Hz}$, arom-H), $7.16(\mathrm{~d}, 2 \mathrm{H}, J=7.2 \mathrm{~Hz}$, arom-H), 7.21-7.37 (m, 2H, arom-H), 7.87-7.97 (m, 8H, arom-H), $10.14(\mathrm{~s}, 1 \mathrm{H}, \mathrm{NH}) ;{ }^{13} \mathrm{C}-\mathrm{NMR}$ (DMSO-d $\left.{ }_{6}\right): \delta=188.41,167.62$ (CO), 146.11, 138.00, 134.68, 131.58, 128.80, 128.44, 126.28, 126.09, 123.26, 114.01, 27.24, $42.80\left(\mathrm{CH}_{2}\right)$; Anal. Calcd. for $\mathrm{C}_{31} \mathrm{H}_{21} \mathrm{~N}_{3} \mathrm{O}_{6}(531.51)$ : C, 70.05; H, 3.98; N, 7.91. Found: C, 70.21; H, 4.20; N, 8.13.

2-(2-3,5-Di[2-(1,3-dioxo-2,3-dihydro-1H-2-isoindolyl)acetyl]phenyl-2-oxoethyl)-1,3-isoindolinedione (9)

Thermal method: A mixture of compound $2(2.58 \mathrm{~g}, 0.01 \mathrm{~mol})$ and acetic acid $(10 \mathrm{~mL})$ was refluxed for $10 \mathrm{~h}$, upon completion of the reaction, as checked by TLC, the product was extracted with EtOH. After evaporation of the solvent under reduced pressure, the product was recrystallized from toluene. This compound was obtained as light brown crystals, yield $(84 \%)$; mp. $300-301{ }^{\circ} \mathrm{C}$; IR $\left(\mathrm{KBr}, \mathrm{cm}^{-1}\right)$ : 1,776 , 1,721, (CO); MS (EI) m/z (\%) = $639\left[\mathrm{M}^{+}\right]$; ${ }^{1} \mathrm{H}-\mathrm{NMR}\left(\mathrm{DMSO}_{6}\right): \delta(\mathrm{ppm}) 5.57\left(\mathrm{~s}, 6 \mathrm{H}, \mathrm{CH}_{2}\right)$, 7.93-7.99 (m-12H, phthalimidyl-H), 8.98 (s, 3H, benzyl-H); ${ }^{13} \mathrm{C}-\mathrm{NMR}$ (DMSO- $d_{6}$ ): $\delta 192.23,167.98$ (2 CO), 135.33, 135.20, 133.39, 132.02, 123.93, $45.50\left(\mathrm{CH}_{2}\right)$; Anal. Calcd. for $\mathrm{C}_{36} \mathrm{H}_{21} \mathrm{~N}_{3} \mathrm{O}_{9}(639.56)$; C, 67.61; H, 3.31; N, 6.57. Found 67.46; H, 3.33; N, 6.81.

Microwave method: A mixture of compound 2 (2.58 g, $0.01 \mathrm{~mol})$, zeolite (Montmorillonite k-10 clay, $1 \mathrm{~g}$ ) and acetic acid $(1 \mathrm{~mL})$ was irradiated in microwave at $150{ }^{\circ} \mathrm{C}$ for $60 \mathrm{~min}$. The crude product was poured onto water, the solid product, so formed, was collected by filtration and crystallized from toluene. This gave compound 9 in $57 \%$ yield.

General procedure for the synthesis of compounds 11a-c

A solution of compound 2 (2.58 g $0.01 \mathrm{~mol})$, compounds 10a-c $(0.01 \mathrm{~mol})$, and EtOH (20 mL) was heated under reflux for $5 \mathrm{~h}$ (completion of reaction was monitored by TLC). The reaction mixture was poured into cold water, filtered and crystallized from ethanol to afford compounds 11a-c.

2-(2-Oxo-4-phenylamino-but-3-enyl)isoindole-1,3-dione (11a): Yellow-orange crystals, yield 88\%; mp 167-169 ${ }^{\circ} \mathrm{C}$; IR $\left(\mathrm{KBr}, \mathrm{cm}^{-1}\right): 3,457(\mathrm{NH}), 1,773,1,706,1,640(\mathrm{CO})$; MS (EI) m/z: (\%) 306 [M+, 20\%]; 160 (25), 146 (100), 117 (8), 104 (14), 77 (12); ${ }^{1} \mathrm{H}-\mathrm{NMR}$ (DMSO-d $)$ ) 4.50 (s, 2H, NCH ), 5.45 (d, 1H, $J=8.0 \mathrm{~Hz}, \mathrm{CH}), 7.03(\mathrm{t}, 1 \mathrm{H}, J=7.2 \mathrm{~Hz}$, phenyl-H), $7.25(\mathrm{~d}, 2 \mathrm{H}, J=8.0 \mathrm{~Hz}$, phenyl-H), $7.30(\mathrm{t}, 2 \mathrm{H}, J$ $=7.2 \mathrm{~Hz}$, phenyl-H), 7.77 (dd, $1 \mathrm{H}, J=8.0 \mathrm{~Hz}, J=8.0 \mathrm{~Hz} \mathrm{CH}), 7.87-7.90$ (m, 2H, phthalimidyl-H), 7.91-7.95 (m, 2H, phthalimidyl-H), $11.15(\mathrm{~d}, 1 \mathrm{H}, J=12.6 \mathrm{~Hz}, \mathrm{NH}) ;{ }^{13} \mathrm{C}-\mathrm{NMR}$ (DMSO- $d_{6}$ ): $\delta=191.47,168.22$ (CO), 145.75, 140.38, 135.16, 132.14, 130.06, 123.96, 123.69, 116.66, 93.58, 45.79 $\left(\mathrm{NCH}_{2}\right)$; Anal. Calcd. for $\mathrm{C}_{18} \mathrm{H}_{14} \mathrm{~N}_{2} \mathrm{O}_{3}$ (306.31): C, 70.58; H, 4.61, N, 9.15. Found: 70.55; H, 4.61, N, 9.27 .

2-[4-(4-Methoxyphenylamino)-2-oxo-but-3-enyl]isoindole-1,3-dione (11b): Yellow crystals, yield 83\%; mp 204-206 ${ }^{\circ} \mathrm{C}$; IR ( $\left.\mathrm{KBr}, \mathrm{cm}^{-1}\right): 3,455(\mathrm{NH}), 1,772,1,706,1,632$ (CO); MS (EI) m/z: (\%) 336 $[\mathrm{M}+, 82 \%] ; 175$ (100), 160 (42), 132 (65), 103 (22), 76 (21); ${ }^{1} \mathrm{H}-\mathrm{NMR}$ (DMSO-d $)$ ) 3.71 (s, 3H, $\left.\mathrm{OCH}_{3}\right), 4.46\left(\mathrm{~s}, 2 \mathrm{H}, \mathrm{NCH}_{2}\right), 5.37(\mathrm{~d}, 1 \mathrm{H}, J=7.8 \mathrm{~Hz}, \mathrm{CH}), 6.88(\mathrm{~d}, 2 \mathrm{H}, J=8.4 \mathrm{~Hz}$, arom-H), 7.19 (d, $2 \mathrm{H}, J=8.4 \mathrm{~Hz}$, arom-H), $7.64(\mathrm{dd}, 1 \mathrm{H}, J=7.8 \mathrm{~Hz}, J=7.8 \mathrm{~Hz} \mathrm{CH}), 7.86-7.89$ (m, 2H, phthalimidyl- 
$\mathrm{H}), 7.91-7.93\left(\mathrm{~m}, 2 \mathrm{H}\right.$, phthalimidyl-H), $11.18(\mathrm{~d}, 1 \mathrm{H}, J=12.6 \mathrm{~Hz}, \mathrm{NH}) ;{ }^{13} \mathrm{C}-\mathrm{NMR}$ (DMSO- $d_{6}$ ): $\delta=190.27,167.70(\mathrm{CO}), 155.80,145.99,134.62,133.30,131.65,123.18,117.65,114.77,92.08,55.99$ $\left(\mathrm{NCH}_{2}\right), 44.34\left(\mathrm{OCH}_{3}\right)$; Anal. Calcd. for $\mathrm{C}_{19} \mathrm{H}_{16} \mathrm{~N}_{2} \mathrm{O}_{4}$ (336.34): C, 67.85; H, 4.79, N, 8.33. Found: C, $67.73 ; \mathrm{H}, 4.85, \mathrm{~N}, 8.30$.

2-[4-(4-Nitrophenylamino)-2-oxo-but-3-enyl]isoindole-1,3-dione (11c): Yellow crystals, yield 79\%; mp 234-236 ${ }^{\circ} \mathrm{C}$; IR (KBr, cm $\left.{ }^{-1}\right): 3,460(\mathrm{NH}), 1,768,1,715,1,653$ (CO); MS (EI) m/z: (\%) 351 [M+, 84\%]; 306 (53), 261 (28), 191 (60), 159 (41), 145 (43), 116 (45), 104 (70). 76 (100); ${ }^{1} \mathrm{H}-\mathrm{NMR}$ $\left(\mathrm{DMSO}_{6}\right)$ ) $4.57\left(\mathrm{~s}, 2 \mathrm{H}, \mathrm{NCH}_{2}\right), 5.65(\mathrm{~d}, 1 \mathrm{H}, J=8.4 \mathrm{~Hz}, \mathrm{CH}), 7.49$ (d, 2H, $J=9.0 \mathrm{~Hz}$, arom-H), 7.82 (dd, $1 \mathrm{H}, J=8.4 \mathrm{~Hz}, J=8.4 \mathrm{~Hz} \mathrm{CH}), 7.85-7.89$ (m, 2H, phthalimidyl-H), 7.90-7.94 (m, 2H, phthalimidyl-H), $8.15\left(\mathrm{~d}, 2 \mathrm{H}, J=9.0 \mathrm{~Hz}\right.$, arom-H), $11.18(\mathrm{~d}, 1 \mathrm{H}, J=12.6 \mathrm{~Hz}, \mathrm{NH}) ;{ }^{13} \mathrm{C}-\mathrm{NMR}$ (DMSO- $d_{6}$ ): $\delta=190.26,167.32$ (CO), 155.72, 145.42, 141.53, 134.57, 131.69, 125.63, 123.32, 116.14, 108.33, $36.55\left(\mathrm{NCH}_{2}\right)$; Anal. Calcd. for $\mathrm{C}_{18} \mathrm{H}_{13} \mathrm{~N}_{3} \mathrm{O}_{5}$ (351.31): C, 61.54; H, 3.73, N, 11.96. Found: C, $61.53 ; \mathrm{H}, 3.82, \mathrm{~N}, 11.96$.

\section{General procedure for the synthesis of $\mathbf{1 2}$ and $\mathbf{1 3}$}

Equimolar quantities $(0.01 \mathrm{~mol})$ of either compound 2 or 11a and corresponding zeolite were heated under solvent-free conditions for $30 \mathrm{~min}$ in a focused microwave oven at $160{ }^{\circ} \mathrm{C}$. Upon completion of the reaction, as checked by TLC, the product was extracted with $\mathrm{EtOH}(3 \times 10 \mathrm{~mL})$. After evaporation of the solvent under reduced pressure, the product was recrystallized from toluene.

2-Phenylisoindole-1,3-dione (12): Buff crystals, yield 96\% ; mp 206-208 ${ }^{\circ} \mathrm{C}$ (lit. [13] mp 205-207 ${ }^{\circ} \mathrm{C}$ yield 95\%); MS (EI) m/z: (\%) 223 [M+, 100\%]; 179 (78), 152 (10), 104 (16), 76 (40). ${ }^{1} \mathrm{H}-\mathrm{NMR}$ (DMSO-d $)_{6}$ ) 7.44 (d, 3H, $J=7.2 \mathrm{~Hz}$, phenyl-H), 7.53 (t, 2H, $J=7.2 \mathrm{~Hz}$, phenyl-H), 7.91-7.94 (m, 2H, phthalimidyl-H), 7.96-7.99 (m, 2H, phthalimidyl-H); ${ }^{13} \mathrm{C}-\mathrm{NMR}$ (DMSO-d ${ }_{6}$ ): $\delta=167.49$ (CO), 135.17, 132.37, 132.02, 129.31, 128.53, 127.89, 123.89; Anal. Calcd. for $\mathrm{C}_{14} \mathrm{H}_{9} \mathrm{NO}_{2}$ (223.23): C, 75.33; $\mathrm{H}$, 4.06, N, 6.27. Found: C, 75.57; H, 4.12, N, 6.36.

2-Methyl-isoindole-1,3-dione (13): Buff crystals, yield 87\%, mp 131-133 ${ }^{\circ} \mathrm{C}$ (lit. [14] mp $134{ }^{\circ} \mathrm{C}$ and yield 68\%); MS (EI) m/z: (\%) 161 [M+, 100\%]; 132 (50), 117 (74), 104 (82), 76 (81), 66 (44); ${ }^{1} \mathrm{H}-\mathrm{NMR}\left(\mathrm{DMSO}-d_{6}\right): 3.36\left(\mathrm{~s}, 3 \mathrm{H}, \mathrm{CH}_{3}\right), 7.81-7.83(\mathrm{~m}, 2 \mathrm{H}$, phthalimidyl-H), 7.84-7.88 (m, 2H, phthalimidyl-H); ${ }^{13} \mathrm{C}-\mathrm{NMR}$ (DMSO- $\left.d_{6}\right): \delta=168.53$ (CO), 134.72, 132.30, 123.36, $24.20\left(\mathrm{CH}_{3}\right)$; Anal. Calcd. for $\mathrm{C}_{9} \mathrm{H}_{7} \mathrm{NO}_{2}$ (161.16): C, 67.07; H, 4.38, N, 8.69. Found: C, 67.01; H, 4.39, N, 9.19.

\section{Reaction of phthalic anhydride with aniline}

Equimolar quantities $(1.48 \mathrm{~g}, 0.01 \mathrm{~mol})$ of phathalic anhydride and aniline $(0.93 \mathrm{~g}, 0.01 \mathrm{~mol})$ were heated for $60 \mathrm{~min}$ in a focused microwave oven at $180{ }^{\circ} \mathrm{C}$ under solvent-free conditions. Upon completion of the reaction, as checked by TLC, the product was extracted with EtOH. After evaporation of the solvent under reduced pressure, the product was recrystallized from toluene to afford compound 12, yield 94\%. 


\section{2-[2-(5-Hydroxynaphtho[1,2-b] furan-3-yl)-2-oxoethyl] isoindole-1,3-dione (18)}

Thermal method: A mixture of compound 11a $(3.06 \mathrm{~g}, 0.01 \mathrm{~mol})$ and naphthoquinone $(1.54 \mathrm{~g}$, $0.01 \mathrm{~mol})$ was dissolved in glacial acetic acid $(10 \mathrm{~mL})$, then stirred overnight at room temperature. The so formed crystals were collected by filtration and crystallized from dioxane. This compound was obtained in $68 \%$ yield, mp $303{ }^{\circ} \mathrm{C}$ (lit. [15] mp 301-303 $\left.{ }^{\circ} \mathrm{C}\right)$; IR $\left(\mathrm{KBr}, \mathrm{cm}^{-1}\right): 3,309(\mathrm{OH}), 1,776$, 1,722, 1,666 (CO); MS (EI) m/z (\%)= $371\left[\mathrm{M}^{+}\right] ;{ }^{1} \mathrm{H}-\mathrm{NMR}\left(\mathrm{DMSO}-d_{6}\right): \delta 5.17\left(\mathrm{~s}, 2 \mathrm{H}, \mathrm{CH}_{2}\right), 7.45(\mathrm{~s}$, $1 \mathrm{H}, \mathrm{H}-4), 7.58$ (dt, 1H, J=7.8, 1.2 Hz, H-7), 7.70 (dt, 1H, J=7.8, 1.2 Hz, H-8), 7.91-7.92 (m, 1H, H14), 7.97-7.98 (m, 1H, H-13), 8.23 (d, 1H, $J=8.4 \mathrm{~Hz}, \mathrm{H}-9), 8.25$ (d, 1H, J=8.4 Hz, H-6), 9.31 (s, 1H, $\mathrm{H}-2), 10.34$ (s, $1 \mathrm{H}, \mathrm{OH}) ;{ }^{13} \mathrm{C}-\mathrm{NMR}$ (DMSO-d $)_{6}$ : $\delta 188.71$ (C-10), 167.99 (C-12), 153.74 (C-2), 151.65 (C-5), 145.02 (C-9b), 135.28 (C-14), 132.02 (C-12a), 128.01 (C-8), 125.61 (C-7), 123.97 (C-5a), 123.89 (C-13), 123.80 (C-6), 121.19 (C-9a), 120.28 (C-3a), 120.08 (C-3), 119.85 (C-9), 100.12 (C-4), $45.23\left(\mathrm{CH}_{2}, \mathrm{C}-11\right)$; Anal. Calcd. for $\mathrm{C}_{22} \mathrm{H}_{13} \mathrm{NO}_{5}$ (371.35); C, 71.16; H, 3.53; N, 3.77. Found C, 71.14; H, 3.65; N, 3.95 .

Microwave method: A mixture of compound 11a $(3.06 \mathrm{~g}, 0.01 \mathrm{~mol})$, naphthoquinone $(1.54 \mathrm{~g}$, $0.01 \mathrm{~mol})$, and glacial acetic acid $(1 \mathrm{~mL})$ was irradiated at $30{ }^{\circ} \mathrm{C}$ for $30 \mathrm{~min}$, continuously at the maximum power of $80 \mathrm{~W}$. After completion of the reaction the solvent was evaporated. The crude product was then collected by filtration and crystallized from dioxane to give the title compound in $76 \%$ yield.

\section{Conclusions}

In summary, we could show that the enaminone $\mathbf{2}$ is a valuable precursor to the tetrahydropyrimidine, dihydropyridine, triacylbenzene and naphthofuran moieties. It is worthwhile to mention here that thermal and microwave assisted reactions were conducted in different solvents with exception of 2, 12 and 13, which were prepared by microwave heating without solvent. We have also revealed that the reactions described proceeded to completion in a much shorter time when irradiated in a focused microwave oven. Moreover, microwave irradiation in combination with simultaneous cooling assisted the reactions and produced somewhat higher yield than those obtained by conventional heating.

\section{Acknowledgments}

The authors are grateful to Kuwait University, Research Administration for financial support through project SC 05/06. Analytical facilities provided by SAF projects \# GS 01/01 \& GS 03/01 are greatly appreciated.

\section{References}

1. Riyadh, S.M.; Abdelhamid, I.A.; Al-Matar, H.M.; Hilmy, N.H.; Elnagdi, M.H. Enamines as precursors to polyfunctional heteroaromatic compounds; a decade of development. Heterocycles 2008, 75, 1849-1905.

2. Stanovnik, B.; Svete, synthesis of heterocycles from alkyl 3-(dimethylamino) propenoates and related enaminones. Chem. Rev. 2004, 104, 2433-2480. 
3. Zhu, S.; Zhao, K.; Su, X.; Ji, S. Microwave-assisted synthesis of new spiro[indoline-3,4'quinoline] derivatives via a one-pot multicomponent reaction. Synth. Commun. 2009, 39, $1355-1366$.

4. Loghmani-Khouzani, H. Sabzyan, H, Rezaei-Pooranari, A. Synthesis and structure of $\alpha$-azo-2ketomethylquinolines. Dye. Pigment. 2008, 76, 447-454.

5. Al-Omran, F.; Abdel Khalik, M.M.; ElKhair, A.A.; Elnagdi, M.H. Studies with functionally substituted heteroaromatics: A novel route for the synthesis of 1-aryl-6-oxopyridazinones, 1arylpyridazine-6-imines and 1-aryl-6-imino-4-pyridazinals. Synthesis 1997, 91-94.

6. Al-Mousawi, S.; Moustafa, M.S.; Elnagdi, M.H. Studies with enamines: Functionally substituted enamines as aldehyde equivalents in Gewald reaction ARKIVOC, 2008, 10, 17-25.

7. Elassar, A.A.; El-Khair, A.A. Recent developments in the chemistry of enaminones. Tetrahedron 2003, 59, 8463-8480.

8. De Koning, C.B.; Michael, J.P.; Riley, D.L. Formal Synthesis of $(5 R, 8 R, 8 \mathrm{a} S)$-indolizidine 209I via enaminones incorporating weinreb amides. Heterocycles 2009, 79, 935-953.

9. Svete, J. Utilisation of chiral enaminones and azomethine imines in the synthesis of functionalised pyrazoles. ARKIVOC 2006, 7, 35-56.

10. Al-Mousawi, S.; John, E.; Al-Kandery, N. Studies with enaminones: Synthesis and chemical reactivity of 2-(4-dimethylamino-2-oxobut-3-enyl)-isoindole-1,3-dione and of 4-(4dimethylamino-2-oxobut-3-enyloxy)-2h-phthalazin-1-one. J. Heterocycl. Chem. 2004, 41, 381-385.

11. Al-Mousawi, S.M.; El-Apasery, M.A.; Al-Kanderi, N.H. Microwave-assisted organic synthesis: the Gabriel approach as a route to new pyrazolylhydrazonoazoles. ARKIVOC 2008, 16, 268-278.

12. Fischer, P.; Schweizer, E.; Langner, J.; Schmidt, U. ${ }^{13} \mathrm{C},{ }^{1} \mathrm{H}$ Long - range coupling constants in configuration assignment of some trisubstituted alkenes. Magn. Reson. Chem. 1994, 32, 567-568

13. Nikpour, F.; Mogaddam, B.M. An expedient method to the synthesis of $n$-substituted $1 h$ isoindole-1,3(2h)-diones. Heterocycles 2008, 75, 2289-2292.

14. Hekmatshoar, R.; Heravi, M.M.; Baghernejad, B.; Asadolah, K. Microwave-assisted N-alkylation of potassium phthalimide and potassium succinimide onto silica gel in dry media. Phos. Sulf. Silic. 2004, 179, 1611-1614.

15. Al-Mousawi, S.M.; El-Apasery, M.A.; Al-Kanderi, N.H. Azolylacetones as precursors to indoles and naphthofurans facilitated by microwave irradiation with simultaneous cooling. Molecules 2009, 14, 2976-2984.

Sample Availability: Samples of compounds 1-18 are available from the authors.

(C) 2010 by the authors; licensee Molecular Diversity Preservation International, Basel, Switzerland. This article is an open-access article distributed under the terms and conditions of the Creative Commons Attribution license (http://creativecommons.org/licenses/by/3.0/). 